\title{
Molecular Mechanism of the Effect of Huanglian Jiedu Decoction on Type 2 Diabetes Mellitus Based on Network Pharmacology and Molecular Docking
}

\author{
Bei Yin $\mathbb{D}^{1},{ }^{1}$ Yi-Ming Bi, ${ }^{1}$ Guan-Jie Fan $\mathbb{D}^{2},{ }^{2}$ and Ya-Qing Xia ${ }^{2}$ \\ ${ }^{1}$ School of Second Clinical Medicine, Guangzhou University of Chinese Medicine, Guangzhou, China \\ ${ }^{2}$ Department of Endocrinology, Guangdong Provincial Hospital of Chinese Medicine, The Second Affiliated Hospital of Guangzhou \\ University of Chinese Medicine, Guangzhou, China
}

Correspondence should be addressed to Guan-Jie Fan; 13925119990@139.com

Received 31 March 2020; Revised 6 July 2020; Accepted 13 July 2020; Published 20 October 2020

Academic Editor: Antonio Brunetti Copyright $\odot 2020$ Bei Yin et al. This is an open access article distributed under the Creative Commons Attribution License, which
permits unrestricted use, distribution, and reproduction in any medium, provided the original work is properly cited.

\begin{abstract}
Background. Huanglian Jiedu Decoction (HLJDD) is a Traditional Chinese Medicine (TCM) formula comprising four herbal medicines. This decoction has long been used in China for clinically treating T2DM. However, the molecular mechanism of HLJDD treat for T2DM is still not fully known. Hence, this study was designed to reveal the synergistic mechanism of HLJDD formula in the treatment of T2DM by using network pharmacology method and molecular docking. Methods. Retrieving and screening of active components of different herbs in HLJDD and corresponding T2DM-related target genes across multiple databases. Subsequently, STRING and Cytoscape were applied to analysis and construct PPI network. In addition, cluster and topological analysis were employed for the analysis of PPI networks. Then, the GO and KEGG enrichment analysis were performed by using ClueGO tool. Finally, the differentially expressed analysis was used to verify whether the expression of key target genes in T2DM and non-T2DM samples was statistically significant, and the binding capacity between active components and key targets was validated by molecular docking using AutoDock. Results. There are 65 active components involved in 197 T2DM-related targets that are identified in HLJDD formula. What is more, 39 key targets (AKT1, IL-6, FOS, VEGFA, CASP3, etc.) and 3 clusters were obtained after topological and cluster analysis. Further, GO and KEGG analysis showed that HLJDD may play an important role in treating T2DM and its complications by synergistically regulating many biological processes and pathways which participated in signaling transduction, inflammatory response, apoptotic process, and vascular processes. Differentially expressed analysis showed that AKT1, IL-6, and FOS were upregulated in T2DM samples and a significant between sample differential expression. These results were validated by molecular docking, which identified 5 high-affinity active components in HLJDD, including quercetin, wogonin, baicalein, kaempferol, and oroxylin A. Conclusion. Our research firstly revealed the basic pharmacological effects and relevant mechanisms of the HLJDD in the treatment of T2DM and its complications. The prediction results might facilitate the development of HLJDD or its active compounds as alternative therapy for T2DM. However, more pharmacological experiments should be performed for verification.
\end{abstract}

\section{Introduction}

Type 2 diabetes mellitus (T2DM) is a metabolic disease caused by impaired insulin secretion or insulin resistance, which induces glucose, fat, and protein metabolism disorders in the body. T2DM is often associated with a high risk of microvascular complications (including retinopathy, nephropathy, and neuropathy) and macrovascular complications (such as cardiovascular complications) [1]. The preva- lence of T2DM is ever-increasing with years [2]. According to statistics, in 2015, 415 million adults aged 20-79 years around the world had diabetes (more than $90 \%$ are T2DM), and China ranked first (109.6 million) [3]. The IDF report estimates that the number of deaths due to diabetes and its complications increased to 5 million during 2015, which is equivalent to one death every six seconds [4]. The epidemic of diabetes and its complications pose a major threat to global health, greatly increasing the burden of death and disability. 




FIgURE 1: Workflow for HLJDD in the treatment of T2DM.

However, the current mainstream treatments for T2DM mainly include diet control, exercise, and oral administration glycemic drugs, and subcutaneous injection of insulin shows limited efficacy. The therapeutic mechanism is focused on a single component, which is effectively control the blood glucose level of patients, but it has no preventive effect on complications. Therefore, based on the advantages of multicomponent and multitarget effects of Traditional Chinese Medicine (TCM), finding a multitarget hypoglycemic drug from TCM resources has gradually become a new research hotspot.

Huanglian Jiedu Decoction (HLJDD) comes from the classic book "The Handbook of Prescriptions for Emergencies" of TCM; it is the first clinical emergency manual in China, which consists of four herbs, namely, Coptidis rhizome, Scutellariae radix, Phellodendri chinensis cortex, and Gardeniae fructus, known as Huanglian (HL), Huangqin (HQ), Huangbo (HB), and Zhizi (ZZ) in Chinese, in the ratio 
TABLE 1: Database and website.

\begin{tabular}{lc}
\hline Database & Website \\
\hline TCMSP & http://lsp.nwu.edu.cn/tcmsp.php \\
Uniprot & http://www.uniprot.org \\
GeneCards & https://www.genecards.org/ \\
OMIM & https://www.omim.org \\
Venny & http://bioinfogp.cnb.csic.es/tools/venny/index.html \\
STRING & https://string-db.org/ \\
Omishare & http://www.omicshare.com/tools/index.php/ \\
RCSB PDB & https://www.rcsb.org \\
PubChem Compound & https://pubchem.ncbi.nlm.nih.gov \\
GEO & https://www.ncbi.nlm.nih.gov/geo/ \\
\hline
\end{tabular}



FIGURE 2: Network of HLJDD's active components. HLJDD formula is consists of HL, HQ, HB, and ZZ. V corresponds to 4 herb nodes. Green, dark blue, red, and purple circles employed to stand for compounds of HL, HQ, HB, and ZZ, respectively. Node size of the compound is proportional to the number of Chinese medicines connected, indicating that different Chinese medicines contain the same chemical components.

of $3: 2: 2: 3$, respectively. It is a heat-clearing agent with the effect of clearing heat and detoxifying. Many scholars have conducted research on the efficacy of HLJDD in the treatment of T2DM. Two meta-analytical papers in China have shown that HLJDD alone is equivalent to metformin in treating T2DM; adding HLJDD to basic hypoglycemic treatment can effectively reduce the levels of Hb1Ac and 2hPG in T2DM patients and can be used to treat early HbAlc $<7.3 \%$ T2DM patients [5, 6]. Zhang et al. found that HLJDD had a significant effect on controlling blood glucose levels and weight and also have a lipid-regulating effect in T2DM rats
[7]. However, due to the complex composition of HLJDD, the relevant mechanism for treating T2DM has not been fully elucidated.

Network pharmacology uses computers, highthroughput omics data analysis, and other technologies to deeply study the pharmacological mechanism of the role of TCM; its research strategy is holistic and systematic, which is consistent with the overall concept of TCM and the principles of dialectical treatment [8]. Our study is the first to use the network pharmacology method to screen the effective bioactive ingredients and targets of HLJDD, analyze its key 




FIGURE 3: Network of active components-targets. 203 human-derived target proteins target nodes represented by light blue hexagon. Green, dark blue, red, and purple circles employed to stand for compounds of HL, HQ, HB, and ZZ, respectively.

targets, and signal pathways for the treatment of T2DM, in order to provide a guideline in the further investigation of this formula. The detailed workflow of this study was showed in Figure 1.

\section{Materials and Methods}

\subsection{Materials. See (Table 1).}

\subsection{Methods}

2.2.1. Collection and Screening of Bioactive Compounds in HLJDD. All candidate herbal compounds of HLJDD were harvested by use of Traditional Chinese Medicine Systems Pharmacology Database and Analysis Platform (TCMSP). Based on literature reports and pharmacokinetic parameters, the pharmacokinetic properties including absorption, distribution, metabolism, and excretion (ADME) are important contributors for bioactivities of drug. As the TCMSP sug- gested, the compounds with oral bioavailability $(\mathrm{OB}) \geq 30 \%$ have good absorption and slow metabolism after oral administration. The compounds with drug - likeness $(\mathrm{DL}) \geq 0.18$ were chemically suitable for drug development. Hence, two ADME-related parameters including $\mathrm{OB} \geq 30 \%$ and $\mathrm{DL} \geq$ 0.18 were employed to identify the potential active compounds in HLJDD [8].

2.2.2. Prediction of Compounds-Related Targets. Thereafter, we undertook a search for targets that corresponding to the active components of HLJDD obtained from the TCMSP. Use UniProt database to convert all target proteins into corresponding gene symbols with "Homo sapiens" species to standardize gene names and organisms, which prevents overannotation of similar proteins [9]. Finally, the information of compound targets was obtained.

2.2.3. Identification of T2DM-Related Targets and Predicting Therapeutic Targets of T2DM. Details on the human genes 
TABLE 2: Active components network information.

\begin{tabular}{|c|c|c|c|c|}
\hline Mol ID & Compound & $\mathrm{OB}(\%)$ & $\mathrm{DL}$ & Herb \\
\hline MOL002915 & Salvigenin & 49.07 & 0.33 & HQ \\
\hline MOL002934 & NEOBAICALEIN & 104.34 & 0.44 & HQ \\
\hline MOL000173 & Wogonin & 30.68 & 0.23 & HQ \\
\hline MOL002937 & DIHYDROOROXYLIN & 66.06 & 0.23 & HQ \\
\hline MOL002927 & Skullcapflavone II & 69.51 & 0.44 & HQ \\
\hline MOL002897 & Epiberberine & 43.09 & 0.78 & HQ, HL \\
\hline MOL002925 & $5,7,2^{\prime}, 6^{\prime}$-Tetrahydroxyflavone & 37.01 & 0.18 & HQ \\
\hline MOL002932 & Panicolin & 76.26 & 0.29 & HQ \\
\hline MOL000228 & (2R)-7-Hydroxy-5-methoxy-2-phenylchroman-4-one & 55.23 & 0.20 & HQ \\
\hline MOL002910 & Carthamidin & 41.15 & 0.24 & HQ \\
\hline MOL002714 & Baicalein & 33.52 & 0.21 & HQ \\
\hline MOL002913 & Dihydrobaicalin_qt & 40.04 & 0.21 & HQ \\
\hline MOL002914 & Eriodyctiol (flavanone) & 41.35 & 0.24 & HQ \\
\hline MOL002928 & Oroxylin a & 41.37 & 0.23 & HQ \\
\hline MOL002933 & $5,7,4^{\prime}$-Trihydroxy-8-methoxyflavone & 36.56 & 0.27 & HQ \\
\hline MOL000449 & Stigmasterol & 43.83 & 0.76 & HQ, ZZ, HB \\
\hline MOL002909 & 5,7,2,5-Tetrahydroxy-8,6-dimethoxyflavone & 33.82 & 0.45 & HQ \\
\hline MOL001689 & Acacetin & 34.97 & 0.24 & HQ \\
\hline MOL001458 & Coptisine & 30.67 & 0.86 & HQ, HL, HB \\
\hline MOL002917 & $5,2^{\prime}, 6^{\prime}$-Trihydroxy-7,8-dimethoxyflavone & 45.05 & 0.33 & HQ \\
\hline MOL000552 & 5,2'-Dihydroxy-6,7,8-trimethoxyflavone & 31.71 & 0.35 & HQ \\
\hline MOL000073 & ent-Epicatechin & 48.96 & 0.24 & HQ \\
\hline MOL000359 & Sitosterol & 36.91 & 0.75 & HQ \\
\hline MOL000358 & Beta-sitosterol & 36.91 & 0.75 & $\mathrm{HQ}, \mathrm{ZZ}, \mathrm{HB}$ \\
\hline MOL012245 & 5,7,4' -Trihydroxy-6-methoxyflavanone & 36.63 & 0.27 & HQ \\
\hline MOL010415 & 11,13-Eicosadienoic acid, methyl ester & 39.28 & 0.23 & HQ \\
\hline MOL008206 & Moslosooflavone & 44.09 & 0.25 & HQ \\
\hline MOL002879 & Diop & 43.59 & 0.39 & HQ \\
\hline MOL012266 & Rivularin & 37.94 & 0.37 & HQ \\
\hline MOL000525 & Norwogonin & 39.40 & 0.21 & HQ \\
\hline MOL001490 & bis[(2S)-2-Ethylhexyl] & 43.59 & 0.35 & HQ \\
\hline MOL002933 & $5,7,4^{\prime}$-Trihydroxy-8-methoxyflavanone & 36.56 & 0.27 & HQ \\
\hline MOL002670 & Cavidine & 35.64 & 0.81 & $\mathrm{HB}$ \\
\hline MOL002666 & Chelerythrine & 34.18 & 0.78 & $\mathrm{HB}$ \\
\hline MOL002663 & Skimmianin & 40.14 & 0.20 & $\mathrm{HB}$ \\
\hline MOL002668 & Worenine & 45.83 & 0.87 & $\mathrm{HB}, \mathrm{HL}$ \\
\hline MOL002662 & Rutaecarpine & 40.30 & 0.60 & $\mathrm{HB}$ \\
\hline MOL005438 & Campesterol & 37.58 & 0.71 & $\mathrm{HB}$ \\
\hline MOL000622 & Magnograndiolide & 63.71 & 0.19 & $\mathrm{HB}, \mathrm{HL}$ \\
\hline MOL001771 & Poriferast-5-en-3beta-ol & 36.91 & 0.75 & $\mathrm{HB}$ \\
\hline MOL002644 & Phellopterin & 40.19 & 0.28 & $\mathrm{HB}$ \\
\hline MOL002643 & Delta 7-stigmastenol & 37.42 & 0.75 & $\mathrm{HB}$ \\
\hline MOL000790 & Isocorypalmine & 35.77 & 0.59 & $\mathrm{HB}$ \\
\hline MOL000787 & Fumarine & 59.26 & 0.83 & $\mathrm{HB}$ \\
\hline MOL001131 & Phellavin_qt & 35.86 & 0.44 & $\mathrm{HB}$ \\
\hline MOL006422 & Thalifendin & 44.41 & 0.73 & $\mathrm{HB}$ \\
\hline MOL001131 & Phellamurin_qt & 56.60 & 0.39 & $\mathrm{HB}$ \\
\hline
\end{tabular}


TABLE 2: Continued.

\begin{tabular}{lcccc}
\hline Mol ID & Compound & OB (\%) & DL & Herb \\
\hline MOL001455 & (S)-Canadine & 53.83 & 0.77 & HB \\
MOL000785 & Palmatine & 64.60 & 0.65 & HB, HL \\
MOL002651 & Dehydrotanshinone II A & 43.76 & 0.40 & HB \\
MOL003095 & 5-Hydroxy-7-methoxy-2-(3,4,5-trimethoxyphenyl) chromone & 51.96 & 0.41 & ZZ \\
MOL001494 & Mandenol & 42.00 & 0.19 & ZZ \\
MOL007245 & 3-Methylkempferol & 60.16 & 0.26 & ZZ \\
MOL001942 & Isoimperatorin & 45.46 & 0.23 & ZZ \\
MOL002883 & Ethyl oleate (NF) & 32.40 & 0.19 & \\
MOL004561 & Sudan III & 84.07 & 0.59 & ZZ \\
MOL001406 & Crocetin & 35.30 & 0.26 & ZZ \\
MOL000422 & Kaempferol & 41.88 & 0.24 & ZZ \\
MOL001941 & Ammidin & 34.55 & 0.22 & ZZ \\
MOL002907 & Corchoroside A_qt & 104.95 & 0.78 & HL \\
MOL002904 & Berlambine & 36.68 & 0.82 & HL \\
MOL002894 & Berberrubine & 35.74 & 0.73 & HL, HB \\
MOL000098 & Quercetin & 46.43 & 0.28 & HL, ZZ, HB \\
MOL001454 & Berberine & 36.86 & 0.78 & HL, HB \\
MOL002903 & (R)-Canadine & 55.37 & 0.77 & HL \\
\hline
\end{tabular}

associated with T2DM were screened from the GeneCards and OMIM database. Using "T2DM" and "type 2 diabetes mellitus" as keywords, and only "Homo sapiens" genes linked to T2DM were acquired. All the target genes obtained above two databases were summarized. Subsequently, active compound targets were mapped to T2DM-related targets, and then, therapeutic targets of HLJDD against T2DM were obtained by using a Venny2.1 online tool.

2.2.4. Protein-Protein Interaction (PPI) Data. The Search Tool for the Retrieval of Interacting Genes (STRING) database provides information on predicted and experimental protein interactions. Enter the therapeutic targets into the String database as a gene symbol. Furthermore, the condition was limited to "Homo sapiens," and the free point was hidden. In the present study, a high confidence score with higher than 0.9 was selected to obtain a PPI data and then save the tsv file for further research.

2.2.5. Network Construction and Analysis. Network construction was performed as follows: (1) the HLJD decoction-active compound network was established by connecting the herbs of HLJDD and their corresponding active compounds; (2) the active compounds-targets network of HLJDD was established by connecting the active compounds and their related targets; (3) a PPI network of therapeutic targets for HLJDD against T2DM was constructed; (4) network of key targets for HLJDD against T2DM by topological analysis was built; (5) clustering modules in the PPI network was built; and (6) decoction-compound-target-pathway network was constructed. All the networks were visualized utilizing by the Cytoscape v3.7.2 software, which is a useful tool for analysis and visualization of molecular interaction networks.
In order to pick out key target genes with high degree of connectivity for HLJDD against T2DM, we use a CytoNCA tool (a plug-in of Cytoscape) to analyze the topological properties of targets. There are four parameters including degree centrality (DC), betweenness centrality (BC), closeness centrality (CC), and eigenvector centrality (EC) were used to estimate the central properties of the nodes in the network. The higher the four quantitative values of a node are, the greater the importance of the node in the network. In the PPI network, DC greater than 2 times the median, BC, CC, and EC are greater than 1 time the median were employed to screen the key targets of HLJDD acting on T2DM.

Targets in different clusters may play similar physiological roles, thus affecting human physiological and pathological processes. To obtain the links between network clusters, a Cluster Marker tool (a plug-in of Cytoscape) was adopted to investigate node composition in the PPI network.

2.2.6. Gene Ontology (GO) Functional Annotation and Kyoto Encyclopedia of Genes and Genomes (KEGG) Pathway Analysis. To elucidate the biological processes of the target proteins in different clusters and their role in signaling transduction, the ClueGO tool (a plug-in of Cytoscape) is used to perform GO functional annotation and KEGG pathway analysis. By entering a list of target gene names and limiting the species to be human, all target gene names are corrected to their official gene symbol. GO terms with $p$ value $<0.01$ and KEGG pathways with $p$ value $<0.01$ corrected by Bonferroni method were considered to have significance. Finally, choosing the first 20 entries and draw them into a bubble chart for visualization by using the online Omishare website.

2.2.7. Differentially Expressed Analysis. The gene expression profiles associated with T2DM were obtained from the Gene 


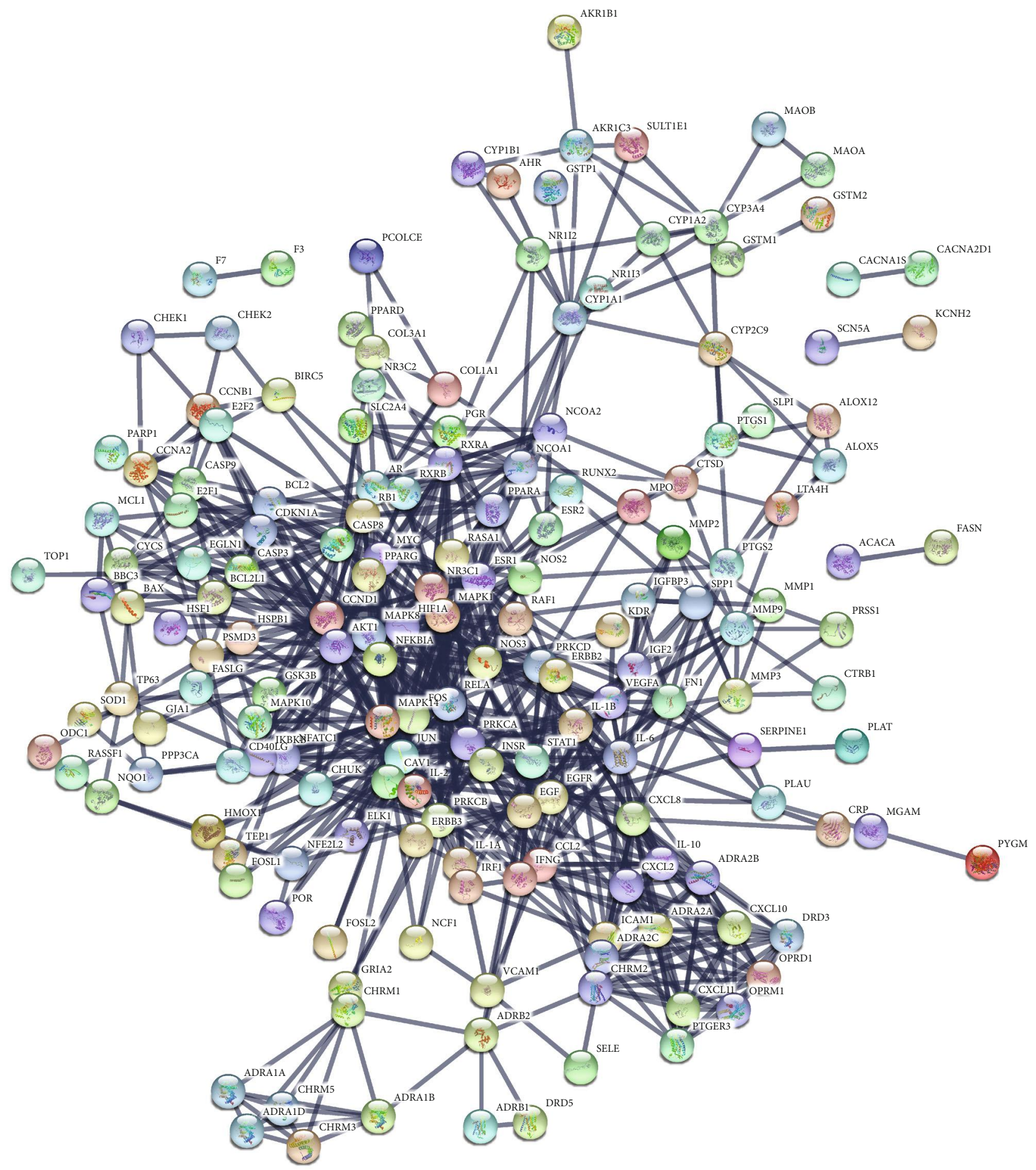

Figure 4: PPI network of targets for HLJDD against T2DM.

Expression Omnibus (GEO) database. The following search terms were used: "Type 2 diabetic or T2DM or Type 2 Diabetes Mellitus," "Homo sapiens," and "Expression profiling by array." After careful screening, the microarray data (GSE29231) submitted by Jain et al. were selected for subsequent analysis [10]. The experimental platform GPL6947 used in the above study was the Illumina HumanHT-12 v3
Expression BeadChip arrays. The dataset included a total of 24 samples: 12 T2DM visceral adipose female samples and 12 non-T2DM visceral adipose female samples. Then, verify whether the targets with highest DC in different clusters are differentially expressed between T2DM and non-T2DM samples using differentially expressed analysis, $P<0.05$ was considered statistically significant. Finally, use the box plots 
TABLE 3: The 39 key targets of HLJDD in treating T2DM.

\begin{tabular}{|c|c|c|c|c|c|}
\hline Target symbol & Target name & $\mathrm{DC}$ & $\mathrm{BC}$ & $\mathrm{CC}$ & EC \\
\hline PTGS2 & Prostaglandin G/H synthase 2 & 91 & 1047 & 0.640 & 0.149 \\
\hline MPO & Myeloperoxidase & 91 & 203 & 0.553 & 0.091 \\
\hline CCL2 & $\mathrm{C}-\mathrm{C}$ motif chemokine 2 & 92 & 261 & 0.595 & 0.130 \\
\hline EGF & Proepidermal growth factor & 92 & 1100 & 0.640 & 0.147 \\
\hline NOS3 & Nitric oxide synthase, endothelial & 91 & 261 & 0.593 & 0.106 \\
\hline IL-10 & Interleukin-10 & 91 & 261 & 0.582 & 0.130 \\
\hline SPP1 & Osteopontin & 91 & 261 & 0.561 & 0.104 \\
\hline EGFR & Epidermal growth factor receptor & 93 & 958 & 0.642 & 0.145 \\
\hline CCND1 & G1/S-specific cyclin-D1 & 92 & 401 & 0.610 & 0.135 \\
\hline CDKN1A & Cyclin-dependent kinase inhibitor 1 & 91 & 261 & 0.556 & 0.135 \\
\hline JUN & Transcription factor AP-1 & 99 & 1005 & 0.655 & 0.158 \\
\hline MAPK14 & Mitogen-activated protein kinase 14 & 91 & 261 & 0.584 & 0.135 \\
\hline HIF1A & Hypoxia-inducible factor 1-alpha & 91 & 261 & 0.562 & 0.135 \\
\hline MMP2 & $72 \mathrm{kDa}$ type IV collagenase & 91 & 261 & 0.586 & 0.135 \\
\hline CASP3 & Caspase-3 & 99 & 1335 & 0.658 & 0.156 \\
\hline HMOX1 & Heme oxygenase 1 & 91 & 261 & 0.567 & 0.135 \\
\hline PPARG & Peroxisome proliferator activated receptor gamma & 91 & 261 & 0.586 & 0.135 \\
\hline IL-2 & Interleukin-2 & 91 & 261 & 0.562 & 0.135 \\
\hline FOS & Proto-oncogene c-Fos & 91 & 1828 & 0.640 & 0.137 \\
\hline SERPINE1 & Plasminogen activator inhibitor 1 & 91 & 261 & 0.566 & 0.135 \\
\hline MAPK8 & Mitogen-activated protein kinase 8 & 95 & 788 & 0.649 & 0.154 \\
\hline FN1 & Fibronectin & 92 & 473 & 0.604 & 0.135 \\
\hline CASP8 & Acacetin & 91 & 261 & 0.564 & 0.135 \\
\hline CYCS & Cytochrome c & 91 & 261 & 0.584 & 0.135 \\
\hline ERBB2 & Receptor tyrosine-protein kinase erbB-2 & 91 & 261 & 0.590 & 0.135 \\
\hline BCL2L1 & Bcl-2-like protein 1 & 91 & 261 & 0.569 & 0.135 \\
\hline AR & Androgen receptor & 91 & 261 & 0.584 & 0.135 \\
\hline MYC & Myc proto-oncogene protein & 94 & 724 & 0.642 & 0.149 \\
\hline MMP9 & Matrix metalloproteinase- 9 & 91 & 1088 & 0.626 & 0.147 \\
\hline MAPK1 & Mitogen-activated protein kinase 1 & 92 & 988 & 0.638 & 0.150 \\
\hline IL-1B & Interleukin-1 beta & 92 & 397 & 0.610 & 0.135 \\
\hline CXCL8 & Interleukin-8 & 91 & 674 & 0.622 & 0.142 \\
\hline ICAM1 & Intercellular adhesion molecule 1 & 91 & 261 & 0.579 & 0.135 \\
\hline VEGFA & Vascular endothelial growth factor A & 105 & 1104 & 0.664 & 0.164 \\
\hline AKT1 & RAC-alpha serine/threonine-protein kinase & 126 & 3090 & 0.729 & 0.174 \\
\hline RELA & Transcription factor p65 & 92 & 261 & 0.588 & 0.135 \\
\hline IL-6 & Interleukin-6 & 110 & 1654 & 0.683 & 0.163 \\
\hline ESR1 & Estrogen receptor & 91 & 741 & 0.616 & 0.131 \\
\hline STAT1 & Signal transducer and activator of transcription 1-alpha/beta & 91 & 261 & 0.567 & 0.135 \\
\hline
\end{tabular}

to show the specific expression of the target genes. Since gene expression profiles are downloaded from GEO (a public database), we do not need to ethically approve, and we do not conduct new experiments on patients or animals.

2.2.8. Component-Target Molecular Docking. AutoDock 4.2.6 is used as molecular docking software for semiflexible docking [11]. The specific operations are as follows: (1) ligand molecule preparation. Determine the compound name, molecular weight, and 2D structure of the compound from the PubChem database. The 3D structure of the compound constructed with the ChemOffice software is saved in * mol2 format. (2) Receptor molecule preparation, select the targets with highest DC in different clusters as the target protein and obtain the 3D structure of the protein receptor from the RCSB PDB database in * PDB format. Use the Pymol 2.3 software to extract the original ligand conformation of the target protein and save it in PDB format. (3) Molecular docking, add polar hydrogen and Gasteiger charge to the processed receptors and ligands with AutoDock Tools (ADT) 
and save the processed receptor and ligand files in PDBQT format. Next, use AutoGrid tool to set the parameters of the docking box (adjust the X-Y-Z coordinates and grid size), apply the Lamarckian genetic algorithm (LGA) to find the best docking conditions for flexible docking, and record the docking position of the receptor and ligand. The analysis of molecular docking results refers to the binding energy ( $\Delta$ Gbind). If the binding energy is less than $-5 \mathrm{~kJ} \cdot \mathrm{mol}^{-1}$, it indicated that the target has certain binding activity with the compound [12]. The lower the binding energy, the better the docking effect. Finally, use the Pymol software to analyze and observe the docking results of compounds and proteins.

\section{Results}

3.1. HLJD Decoction-Active Compounds Network Analysis. By searching the TCMSP databases, the compounds contained in $\mathrm{HL}, \mathrm{HQ}, \mathrm{HB}$, and $\mathrm{ZZ}$ are $48,143,140$, and 98, respectively. According to the characteristics of $\mathrm{OB}$ and $\mathrm{DL}$ of the compounds, $14,36,37$, and 15 active ingredients were screened out, respectively. There are 89 nodes ( 84 compound nodes, 4 herb nodes, and 1 formula nodes) and 105 edges composed HLJD decoction-active compounds network (Figure 2). In this network, coptisine and berberine exists in HL, HQ, and HB. Quercetin exists in HL, HB, and ZZ. Stigmasterol and beta-sitosterol exist in HQ, HB, and ZZ. Supraene exists in HQ and ZZ. Berberrubine, palmidin A, magnograndiolide, worenine, and obacunone exist in HL and $\mathrm{HB}$. The results indicate that the same components may exist in multiple Chinese medicines, and different medicines contain multiple effective components which is an important material basis for the multiple target effects of TCM.

3.2. Active Compounds-Targets Analysis. The corresponding targets of HL, HQ, HB, and ZZ were 133, 88, 55, and 170, respectively. After removing duplicate targets, a total of 203 human-derived target proteins were obtained. The active compounds- targets network of HLJDD is shown in Figure 3. The network consists of 268 nodes (203 target nodes and 66 compound nodes) and 1074 edges, where the nodes represent the active compounds of herbs and compound-related targets, and the edges represent the interaction between the active compounds and the target protein. Among these targets, a total of 135 targets involved in quercetin, 51 targets related to wogonin and kaempferol, and 29 targets involved in baicalein. Other active compounds also have more corresponding targets, such as isocorypalmine, beta-sitosterol, and stigmasterol, all correspond to 26 targets. This suggests that compounds in the HLJDD formula may work together on these targets, exerting pharmacological effects in T2DM and other diseases.

3.3. PPI Network of Therapeutic Targets for HLJDD against T2DM. From the GeneCards and OMIM database, 11,230 T2DM-related targets were acquired. A total of 65 active components (Table 2) and 197 overlapping genes were obtained by looking for the intersection of the 203 compound-related targets and the 11,230 T2DM-related

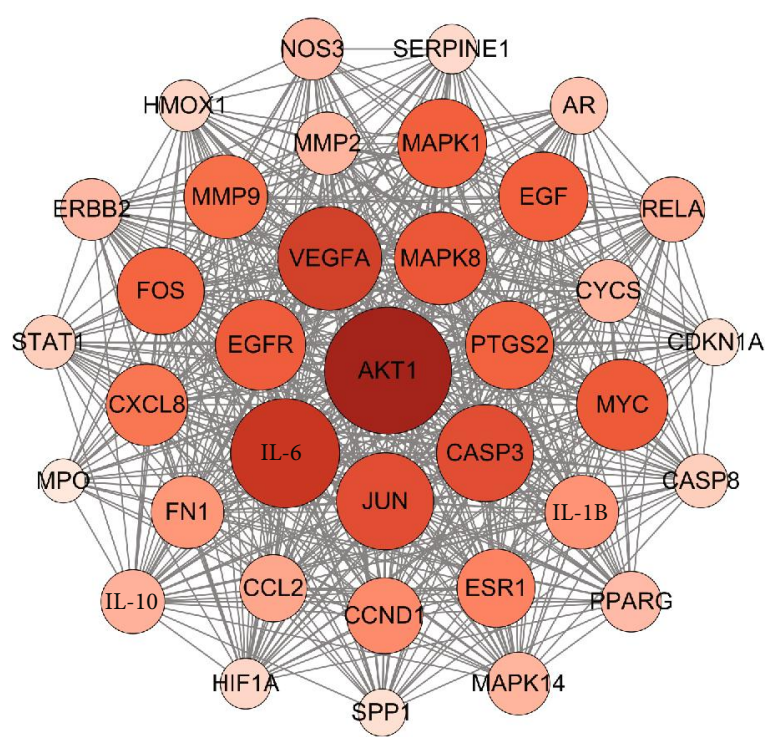

Figure 5: Key targets of HLJDD against T2DM.

targets by using Venny2.1 online tool. The 197 overlapping genes were submitted to the STRING database. At the same time, hide the free point (DCAF5 and DIO1). Finally, 195 target genes with higher degree (degree $\geq 0.700$ ) of connectivity in PPI network were obtained (Figure 4). Download the tsv file in STRING. Then, the tsv file was used to analyzed PPI network by using Cytoscape v3.7.2.

3.4. Topological Analysis and Cluster Analysis. The central properties of each node in PPI network were estimated by topological analysis. According to the network pharmacology method, based on criteria of $\mathrm{DC} \geq 52, \mathrm{BC} \geq 71.46, \mathrm{CC} \geq 0.50$, and $\mathrm{EC} \geq 0.04,39$ key targets of HLJDD acting on T2DM were obtained (Table 3). In Figure 5, the larger of nodes was proportional to DC. Notably, AKT serine/threonine kinase $1($ AKT1, degree $=126)$ which played an essential role in the pathogenesis of T2DM was identified as the most important target in the PPI network. With regard to the target, some nodes (IL-6, VEGFA, MAPK8, JUN, CASP3, EGFR, PTGS2, and FOS) also have higher degrees, which were also recognized as important T2DM targets.

Community cluster (Cluster Marker tool) is a complex algorithm based on clustering objects with similar attributes. Target gene information in PPI network obtained from the STRING database was analyzed by Cluster Marker tool returned 3 central gene clusters. Clusters 1, 2, and 3 involved 70,67 , and 58 target proteins, respectively. In PPI network of target genes based on the results of cluster analysis and topology analysis, key targets are shown in red (Figure 6).

3.5. GO Functional Annotation Analysis. ClueGO tool was used to perform GO and KEGG enrichment analyses on target genes in the above mentioned three modules. We studied the role of clusters 1,2, and 3 in gene function and obtained 123,137 , and $119 \mathrm{GO}$ entries, respectively, which were primarily involved in cell response to different stimuli, inflammatory response, regulation of apoptotic process, and 


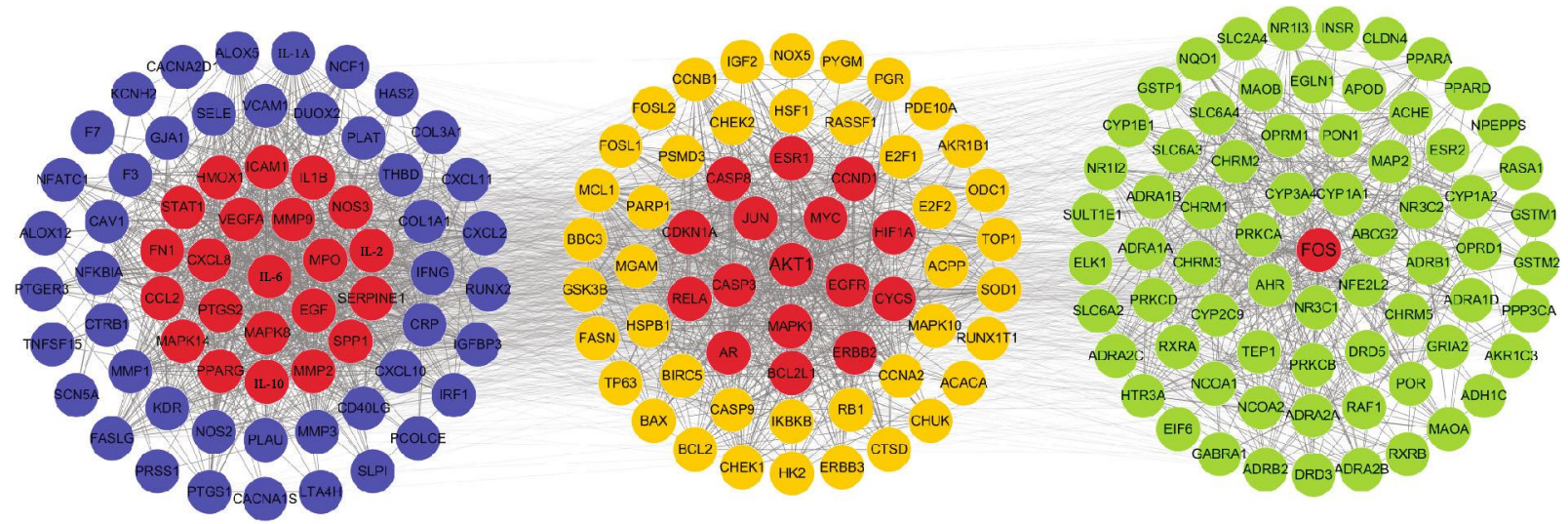

Figure 6: PPI network of target genes based in different cluster. Clusters 1, 2, and 3 are represented by dark blue, orange, and green, respectively. Red nodes represent the key targets. And the lines among nodes display the relationship between different target genes.

regulation of apoptotic-related pathways, receptor activity, and vascular processes (such as angiogenesis, vasculature development, endothelial cell proliferation, nitric oxide biosynthesis, regulation of arterial blood pressure, vessel diameter, and vascular processes). The top 20 entries $(P<0.01)$ were selected based on $p$ value and number of genes, displayed in Figure 7.

3.6. KEGG Pathway Analysis. KEGG pathway analysis shows that targets of different clusters were primarily distributed in pathways are related to diabetes and its complications. The representative top 20 pathways based on the number of enriched genes as well as $p$ value are shown in Figure 8. Of these, cluster 1 mainly included AGE-RAGE signaling pathway in diabetic complications $\left(p=5.30 \mathrm{E}^{-25}\right)$, fluid shear stress and atherosclerosis $\left(p=7.46 \mathrm{E}^{-19}\right)$, TNF signaling pathway $\left(p=6.04 \mathrm{E}^{-15}\right)$, and IL-17 signaling pathway $\left(p=1.30 \mathrm{E}^{-14}\right)$. Module 2 focuses on apoptosis $\left(p=9.71 \mathrm{E}^{-19}\right)$ and tumors pathways, including pancreatic cancer $\left(p=1.07 \mathrm{E}^{-18}\right)$ and colorectal cancer $\left(p=6.55 \mathrm{E}^{-18}\right)$. It is also involved in viral infections, such as hepatitis $\mathrm{B}\left(p=9.71 \mathrm{E}^{-19}\right)$, EB virus $\left(p=2.25 \mathrm{E}^{-17}\right)$, and hepatitis $\mathrm{C}\left(p=8.18 \mathrm{E}^{-17}\right)$. In addition, endocrine resistance $\left(p=1.85 \mathrm{E}^{-15}\right)$, insulin signaling pathway $\left(p=1.04 \mathrm{E}^{-06}\right)$, insulin resistance $\left(p=1.91 \mathrm{E}^{-04}\right)$, type II diabetes $\left(p=5.23 \mathrm{E}^{-04}\right)$, and other signaling pathways also involved. Module 3 is related to neuroactive ligand-receptor interaction $\left(p=7.44 \mathrm{E}^{-10}\right)$ and calcium signaling pathway $\left(p=1.63 \mathrm{E}^{-07}\right)$, as well as the establishment of synapses, such as dopaminergic synapse $\left(p=1.22 \mathrm{E}^{-06}\right)$, cholinergic synapse $\left(p=3.69 \mathrm{E}^{-05}\right)$, and serotonergic synapse $\left(p=4.10 \mathrm{E}^{-05}\right)$.

\subsection{Differentially Expressed Analysis of AKT1, IL-6, and FOS} in Human Type 2 Diabetic Visceral Adipose. Gene expression data were downloaded from the GEO database, including 12 T2DM samples and 12 nondiabetic visceral adipose female controls from GSE29231. Differentially expressed analysis showed that AKT1, IL-6, and FOS were upregulated in T2DM samples, and a significant between sample differential expression with a $p$-value $<0.05$. The results of gene expres- sion in T2DM and nondiabetic samples are shown in Figure 9.

3.8. Validation of Compound-Target Interaction by Molecular Docking. To further validate the binding capacity between active compounds and key targets and improving the accuracy of target network, molecular docking through AutoDock was performed. Molecular docking analyzed three targets (AKT1, IL-6, and FOS) with highest DC in different clusters and 5 compounds (quercetin, wogonin, baicalein, kaempferol, oroxylin A) obtained from active componentstargets network. The results are shown in Table 4 . The binding energy values of most of them were smaller than $-5 \mathrm{~kJ} \bullet \mathrm{mol}^{-1}$, which showed that they possessed good binding activity.

3.9. Integrated Network Construction. To shed light on the potential mechanisms of HLJD decoction acting on T2DM, we constructed a decoction-compound-target-pathway network (Figure 10). The blue line is mainly connected to the diabetic vascular disease-related pathway-the pathway with the highest significance of $p$ value and its key targetcomponent complex with the highest binding energy values. The red line is connected to the T2DM, cancer, and diabetes cognitive dysfunction-related pathway-its key targetcomponent complex with the highest binding energy values. The orange line is linked to the key target in the diabetes cognitive dysfunction-related pathway and the key targetcomponent complex with the highest binding energy values. As observed in the results of network analysis, our research directly confirmed that multitarget of four herbs in HLJD decoction act synergistically in treating T2DM and its complications.

\section{Discussion}

Diabetes belongs to the category of "Xiao Ke" in TCM. It is roughly divided into three types, such as yin deficiency and dryness type, qi and yin deficiency type, and yin and yang deficiency type. Bitter-taste Chinese materia medica (CMM) is mainly used for the treatment of diabetes in yin 


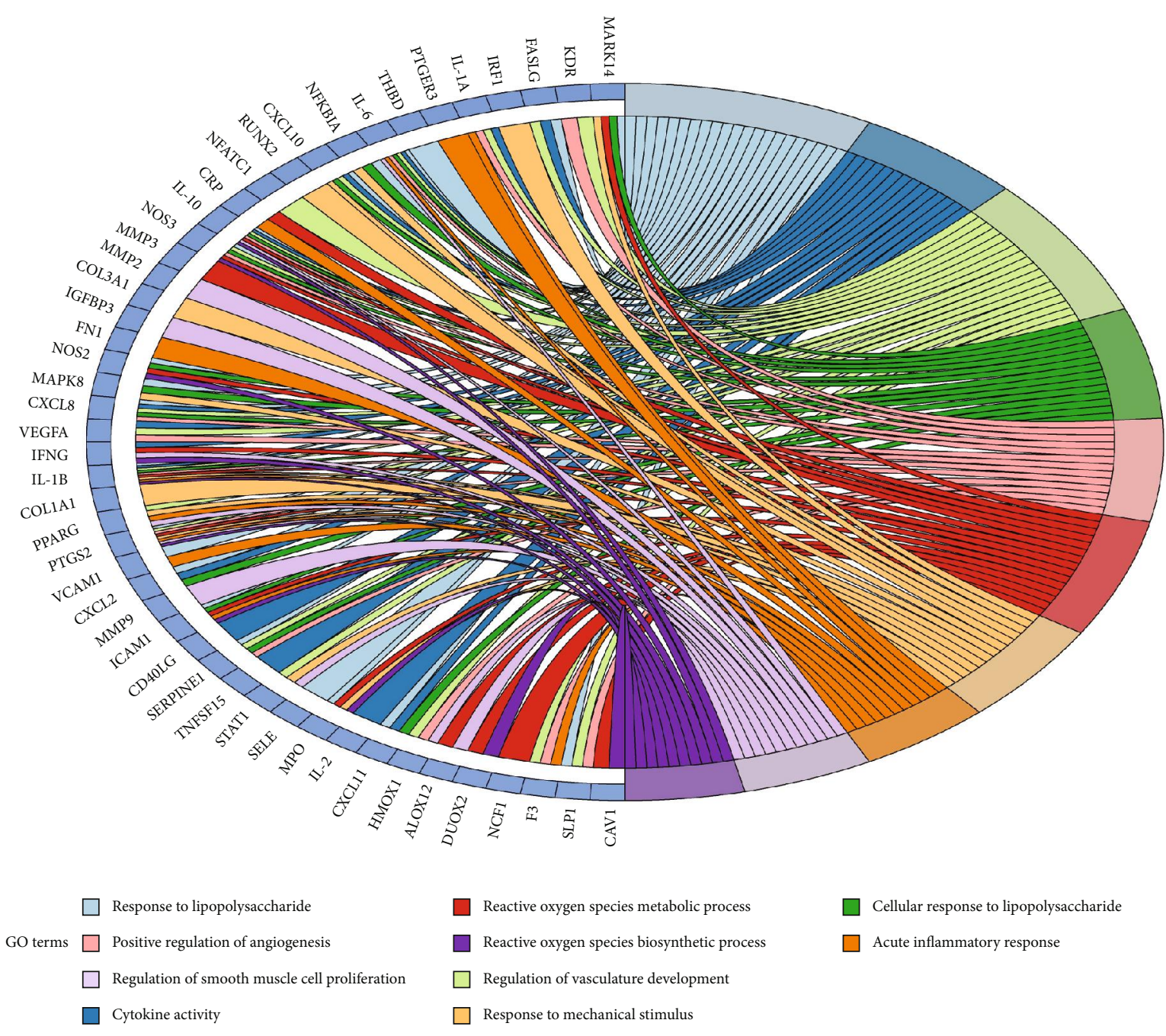

(a)

FIGURE 7: Continued. 


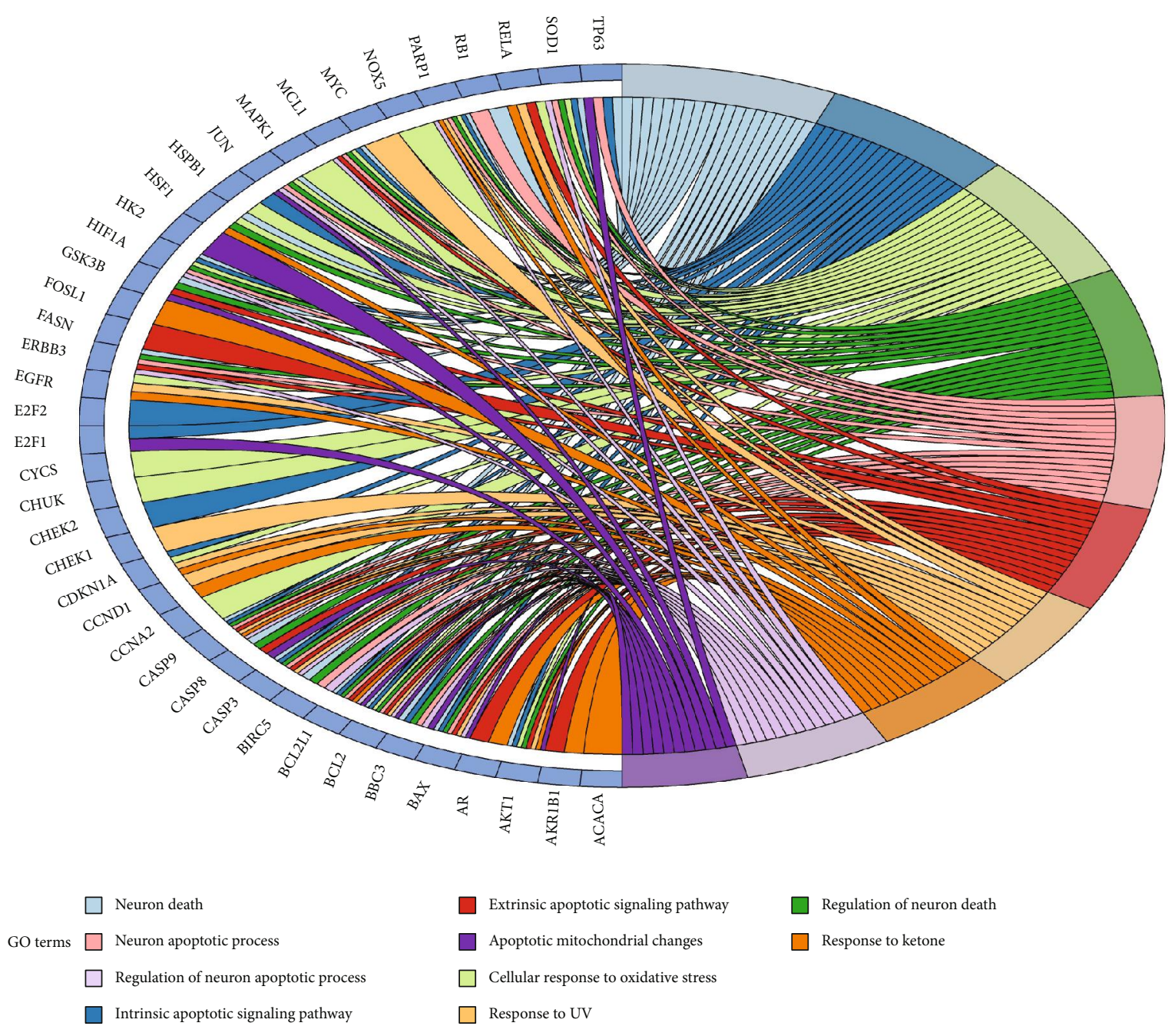

(b)

Figure 7: Continued. 


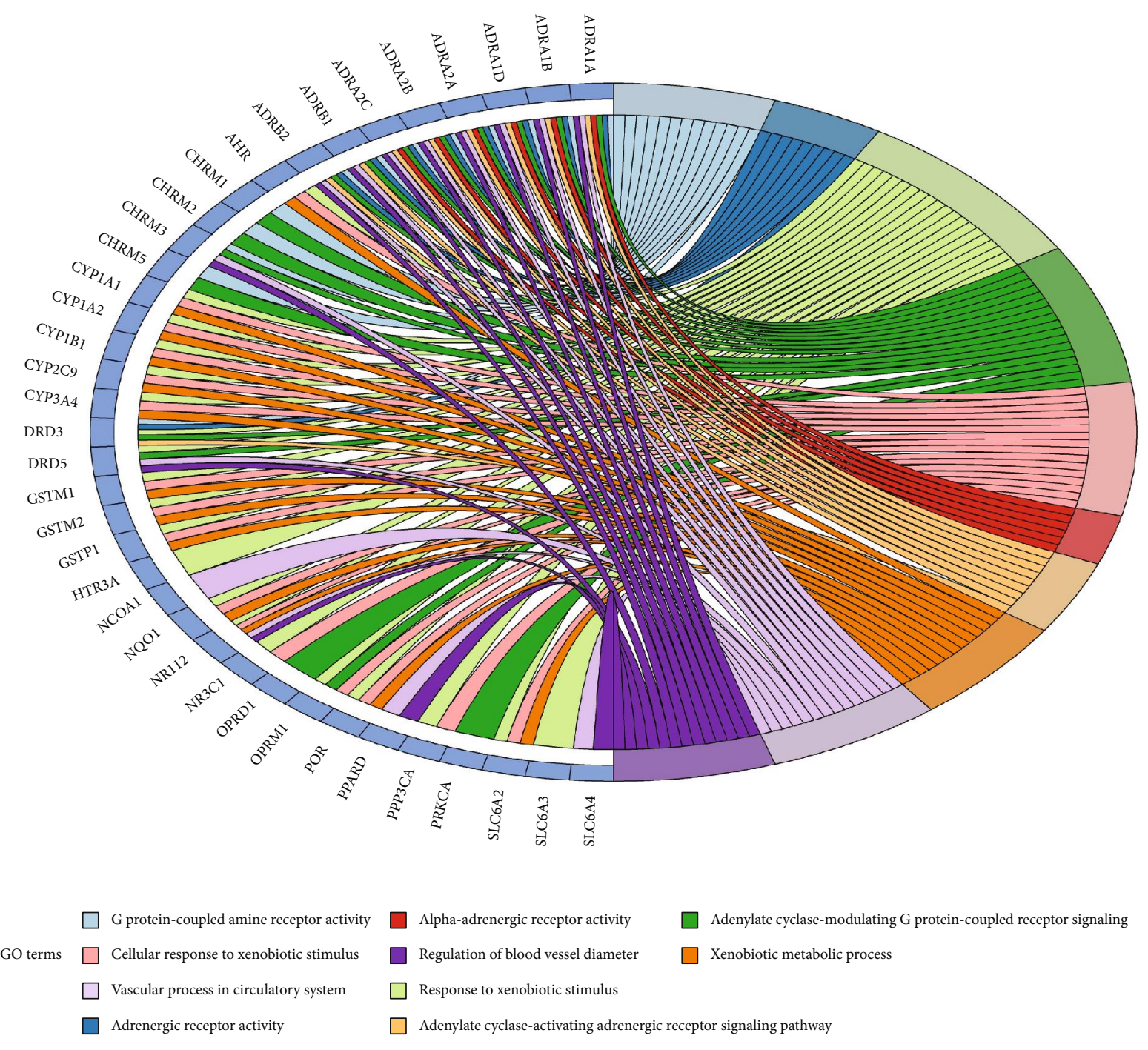

(c)

Figure 7: (a) GO biological process analysis of cluster 1. (b) GO biological process analysis of cluster 2. (c) GO biological process analysis of cluster 3 .

deficiency and heat type [13]. In ancient times, there was a saying that "bitter can restrict sweetness," and there are records in ancient books of using CMM to treat diabetes, because "strengthening Yin with bitter-flavor herbs," which means that some CMMs can protect the yin by reducing heat [14]. HL, HQ, HB, and ZZ in HLJDD formula are all CMM and have the effect of clearing away heat. According to the published literature, HLJDD has exact prevention and treatment effect on diabetes and its complications [15-18].

In the present study, we applied the systematic pharmacological method to predict and elucidate the potential molecular mechanisms of action of the HLJDD on T2DM. In the HLJDD's active components-targets network, a total of 203 targets affected by 66 bioactive compounds in the HLJDD were obtained. There were 4 compounds including quercetin, wogonin, kaempferol, and baicalein identified as the potential active ingredients of HLJDD, of which the biological activities against T2DM were reported previously. For example, quercetin and kaempferol, wogonin, and baicalein, that is, are flavonoid compounds with a similar mechanism of action as metformin, which could reduce glucose and improve glucose Glut4 and AMPK expression in skeletal muscle and adipose tissue, thereby increasing insulin sensitivity and improvement of islet $\beta$ cell quality [19-21]. Alkhalidy illustrated that kaempferol can increase AKT and hexokinase activity, reduce the activity of pyruvate carboxylase and glucose- 6 phosphatase in the liver, and exert antidiabetic effects by inhibiting gluconeogenesis in the liver [22]. In addition, kaempferol also can reduce myocardial ischemiareperfusion injury/MAPK-induced oxidative stress and inflammation by reducing AGE-RAGE, thereby improving myocardial injury in diabetic rats [23]. Yang discovered that baicalein $\left(10^{-6}\right.$ and $\left.10^{-5} \mathrm{~mol} / \mathrm{L}\right)$ may promote glucose uptake and glycolysis through the InsR/IRS-1/PI3K/AKT pathway and inhibit gluconeogenesis in liver cells, thus have strong anti-IR hepatocyte activity [24]. Therefore, the above active components indicate the effectiveness and diversity of chemical ingredients in HLJDD for treating T2DM. In addition, 


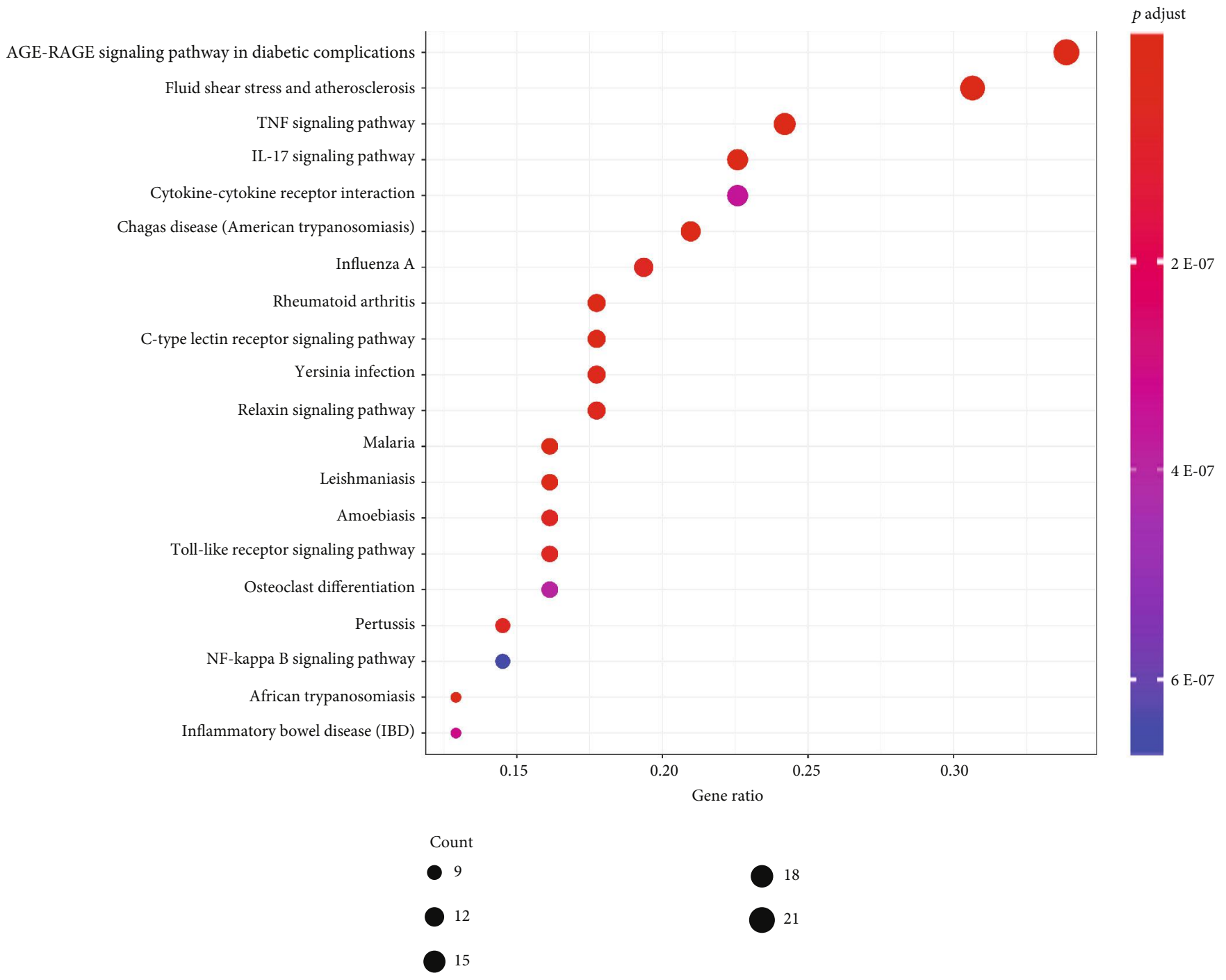

(a)

Figure 8: Continued. 


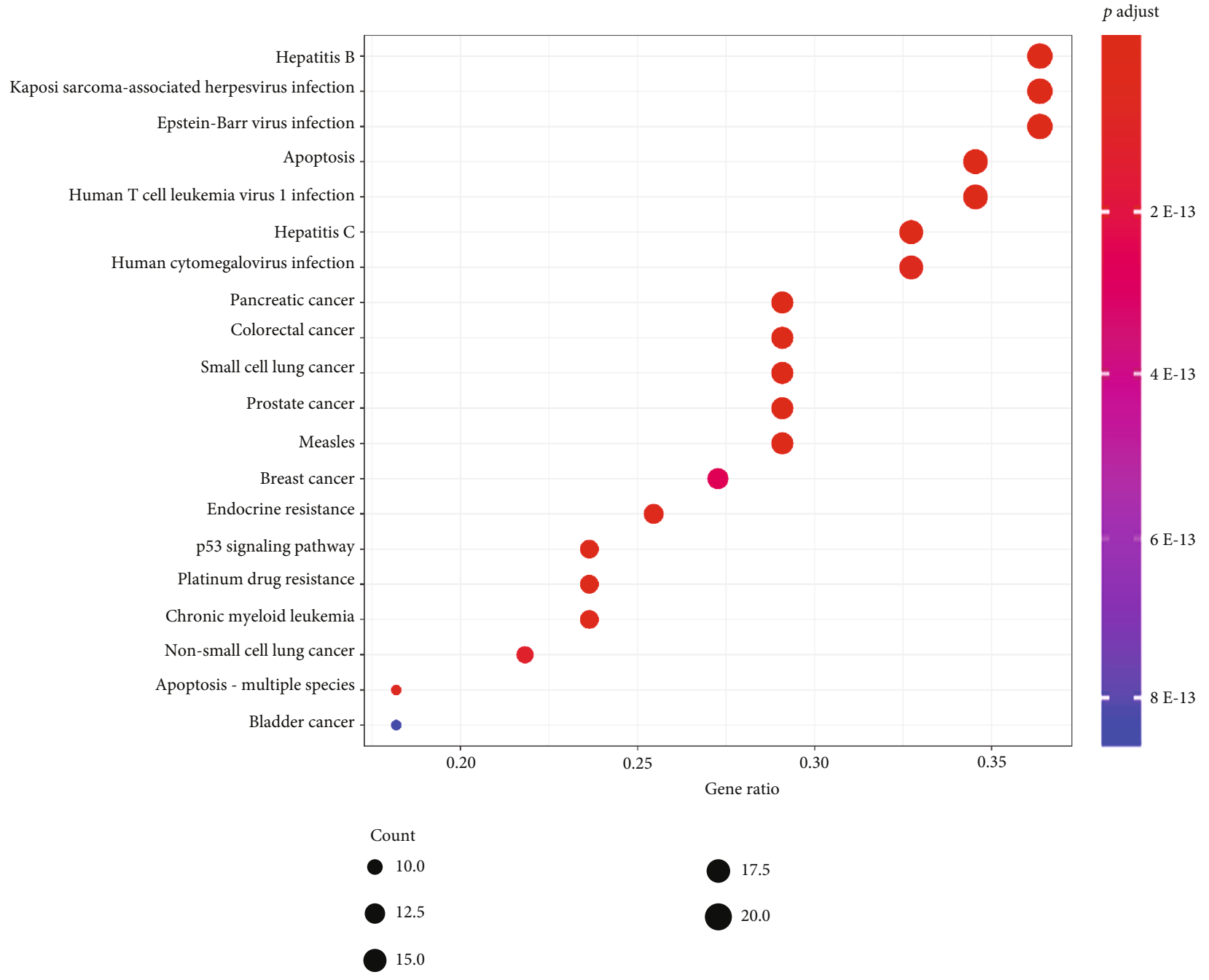

(b)

FIgURe 8: Continued. 


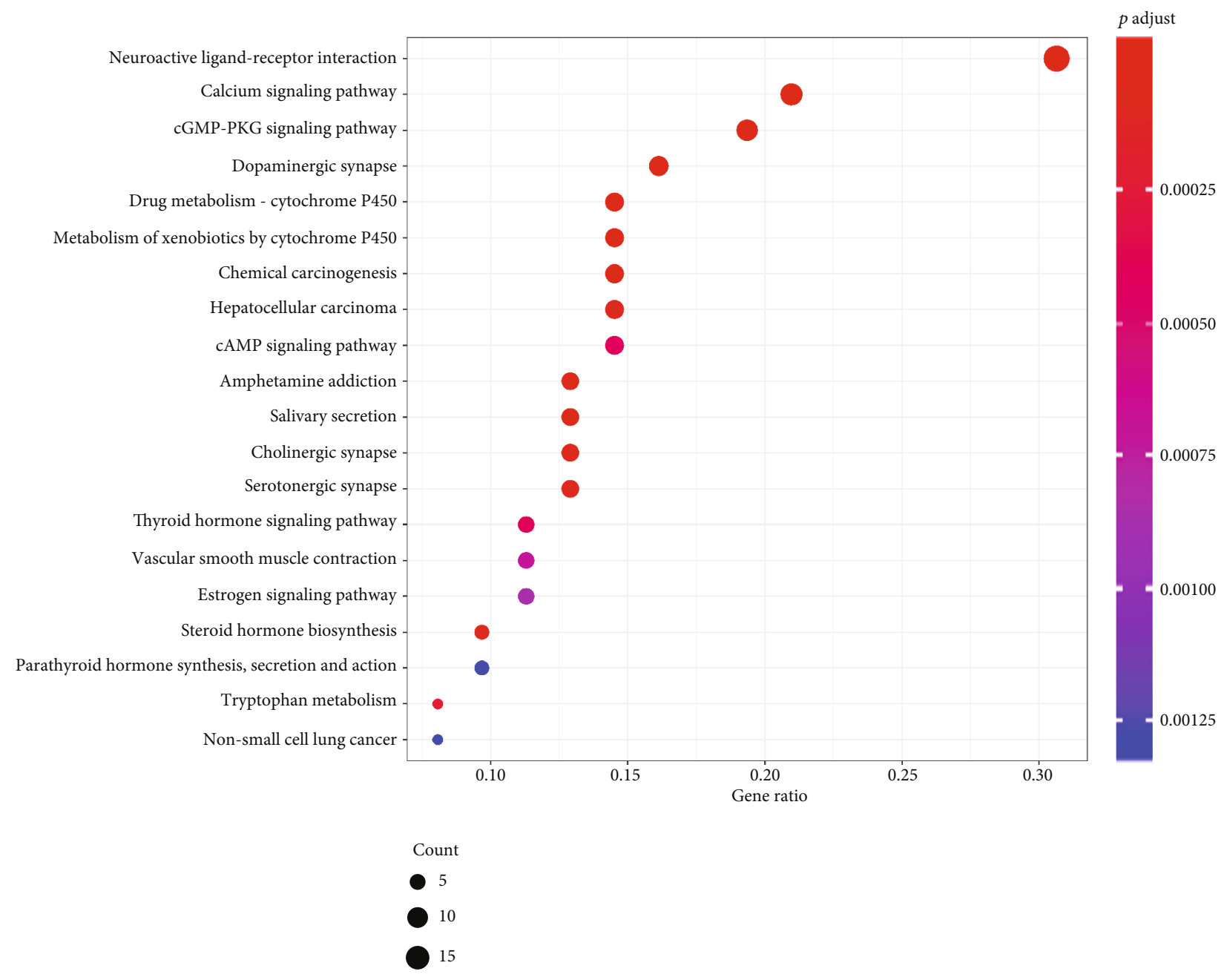

(c)

FIGURE 8: (a) KEGG pathway analysis of cluster 1. (b) KEGG pathway analysis of cluster 2. (c) KEGG pathway analysis of cluster 3.

oroxylin A also plays an important role in the network, oroxylin A possessing a broad spectrum of pharmacological effects, especially anticancer, anti-inflammatory, and neuroprotective activity [25], but there is relatively little research on its pharmacological treatment of T2DM, which deserves further discussion.

The results of PPI network analysis acquired 197 targets of HLJDD acting on T2DM. The GO enrichment analysis of 197 targets indicates that the therapeutic effect of the HLJDD in T2DM mainly involved in biological processes such as cell response to different stimuli, regulation of transcription, inflammatory response, regulation of apoptotic process and regulation of apoptotic-related pathways, receptor activity, vascular processes, and regulation of gene expression, via molecular function of protein binding. Apoptosis plays important roles in the pathophysiology of T2DM [26]. Rehab observed that the STZ-induced diabetes significantly increased the expression of the apoptosis biomarker C-fos gene [27]. Experimental studies have also confirmed that MYC, CASP3, JUN, and EGFR are related to islet $\beta$-cell apoptosis [28], while AKT1 acts as an antiapoptotic signaling kinase in a variety of cells. Zhang Rui found that increasing the expression of Akt in islet cells can reduce islet cell apoptosis and increase secretory function [29]. In addition, $\mathrm{Xu}$ have found that AKT1 gene was activated $\mathrm{CpG}$ island demethylation in the promoter of the AKT1 gene is involved in the occurrence of cardiovascular and cerebrovascular complications in T2DM [30]. IL-6 has been identified as a key mediator of inflammation, immune response, and glucose metabolism; it is the basis of insulin resistance in patients with T2DM [31]. Wu et al. discovered that tocilizumab (an IL-6 receptor antibody) inhibits the activation of NLRP3 inflammasome by inhibiting IL-17A, improves insulin resistance, and has protective effects on diabetic kidney injury [32]. In addition to being directly related to T2DM, the polymorphisms of VEGFA, EGFR, PTGS2, and AKT1 genes are mainly related to diabetic vascular dysfunction and play an important role in diabetic vascular disease and diabetic nephropathy [33-36].

As observed in the results of KEGG enrichment analysis, some signaling pathways (such as AGE-RAGE signaling pathway in diabetic, IL-17signaling pathway, TNF signaling pathway, Toll-like receptor signaling pathway, type II diabetes, endocrine resistance, and insulin resistance signaling 




Group

追 Diabetic

追 Nondiabetic

(a)
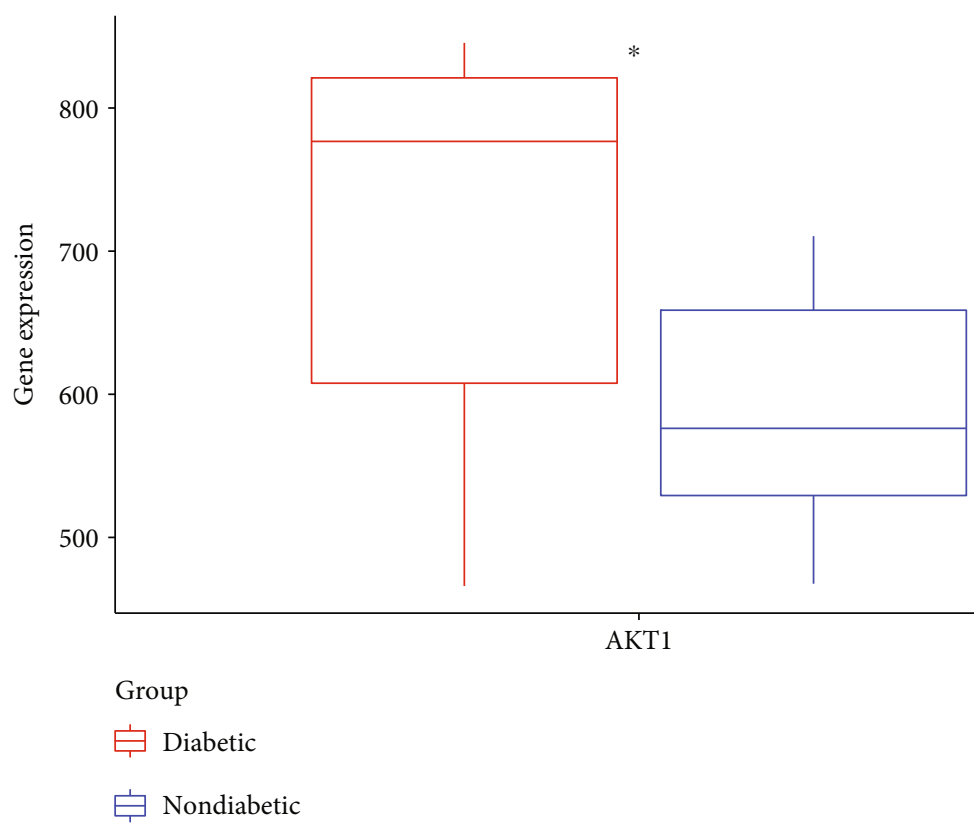

AKT1

Group

追 Nondiabetic

(b)

Figure 9: Continued. 


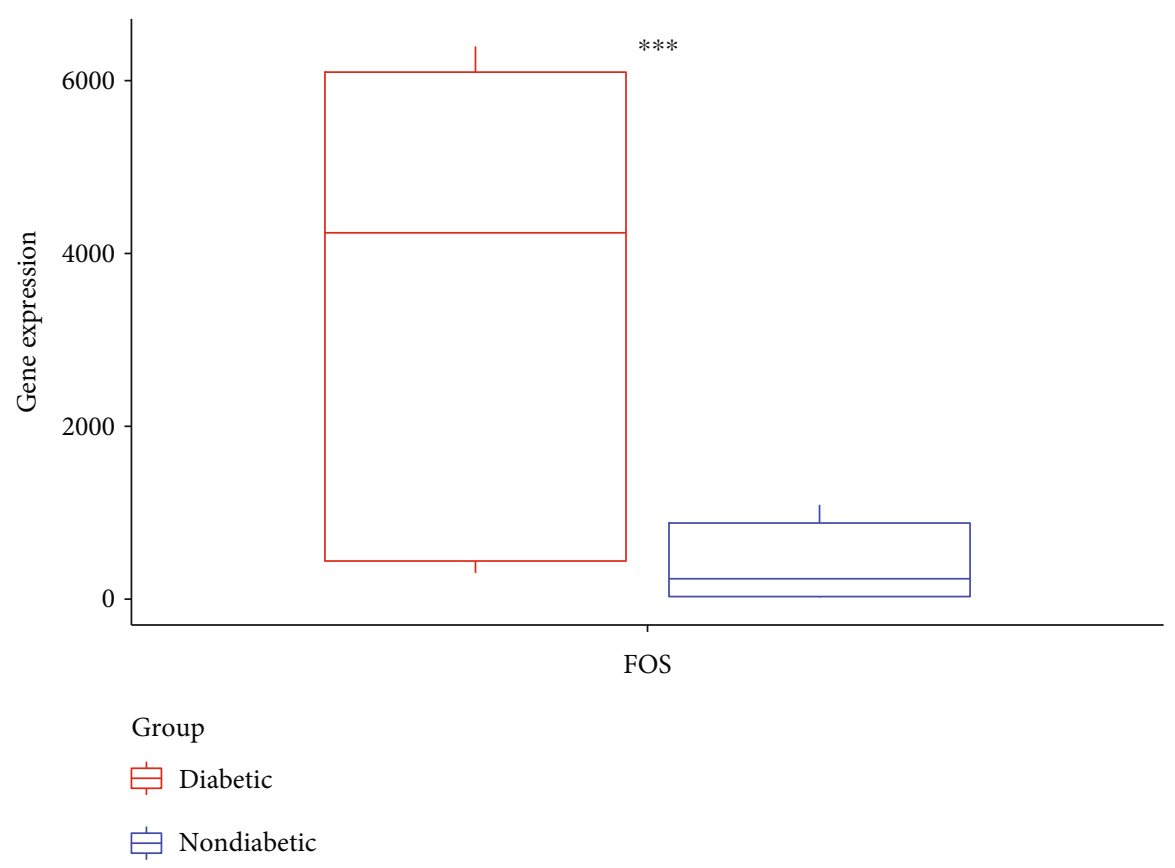

(c)

Figure 9: (a) IL-6. (b) AKT1. (c) FOS.

pathways) are closely related to the development and metastasis of T2DM. Increasing evidence indicates that chronic hyperglycemia leads to the formation of AGEs, and AGERAGE activation leads to oxidative stress and inflammation associated with cardiovascular complications of diabetes $[23,37]$. There is also accumulation of evidence that AGE/RAGE interacts with TNF signaling pathway, perhaps even through mutual amplification to induce the production of superoxide in T2DM [38]. Fluid shear stress and flow pattern also play an important role in atherosclerosis [39]. Deng et al. found that HLJDD can significantly reduce blood glucose and glycosylated hemoglobin $(\mathrm{GH})$ in diabetic rats and inhibit the generation of AGEs in plasma and kidney tissues $(p<0.01)[40]$.

Some experts had put forward some hypothesis that insulin resistance, inflammation, accumulation of advanced glycation end products (AGEs), and oxidative stress may be associated with an increased risk of dementia in the T2DM population [41, 42]. It is reported that HLJDD plays an important role in the mechanism of neuronal injury through neuroinflammation-related signaling pathways, such as MAPK, Toll-like receptors, and JNK/SAPK9 pathways, what is more, it can also act on the insulin signaling pathway to clear/reduce $\mathrm{A} \beta$ in the brain and inhibit Tau protein hyperphosphorylation [43]. HLJDD can improve diabetes cognitive dysfunction by regulating neuroinflammation-related signaling pathways in cluster 2. Acetylcholine was significantly reduced in diabetic patients [44]. It is found that HLJDD has effects on neurotransmitters and regulatory pathways such as cholinergic, serotonergic, and dopaminergic synapses in cluster 3 .

In cardiomyocytes, $\mathrm{Ca}^{2+}$ is the core of excitationcontraction coupling and affects various signal cascades
[45]. In the pathogenesis of diabetic cardiomyopathy, AGEs stimulated the increase of $\mathrm{Ca}^{2+}$ and reduces MMP, which eventually damages myocardial contractile and diastolic functions, and induces apoptosis [46]. HLJDD prevents and treats diabetic cardiomyopathy via calcium signaling pathway in cardiomyocytes in cluster 3 .

Many studies supporting that hyperglycemia, insulin resistance, hyperinsulinemia, IGF-1 levels, dyslipidemia, inflammatory, and cytokines affect tumor cell microenvironment and intracellular signal transduction and help tumor growth and progression [47]. The overall assessment of many large-scale epidemiological studies and metaanalyses has shown that the incidence of cancer at specific sites in T2DM patients continues to increase, including pancreatic cancer, hepatobiliary cancer, breast cancer, and colorectal cancer [47]. HLJDD exhibits antitumor effect involved in regulating multiple tumor-related pathways in cluster 2 .

The top degrees of the potential target genes in the three clusters for the HLJDD in the treatment of T2DM by cluster and topological analysis include AKT1, IL-6, and FOS. We compared the gene expression levels of AKT1, IL-6, and FOS between diabetic patients and nondiabetic and the statistical relationship. The analysis results of differential expression verified that AKT1, IL-6, and FOS were upregulated in T2DM samples, and the difference between the samples was significant $(p<0.05)$. Further molecular docking assay in this study showed that quercetin was verified strong binding activity with AKT1, IL-6, and FOS, while baicalein had strong combination with AKT1 and FOS, wogonin had strong combination with AKT1 and IL-6, kaempferol had strong combination with AKT1, and oroxylin A had strong combination with IL-6. 


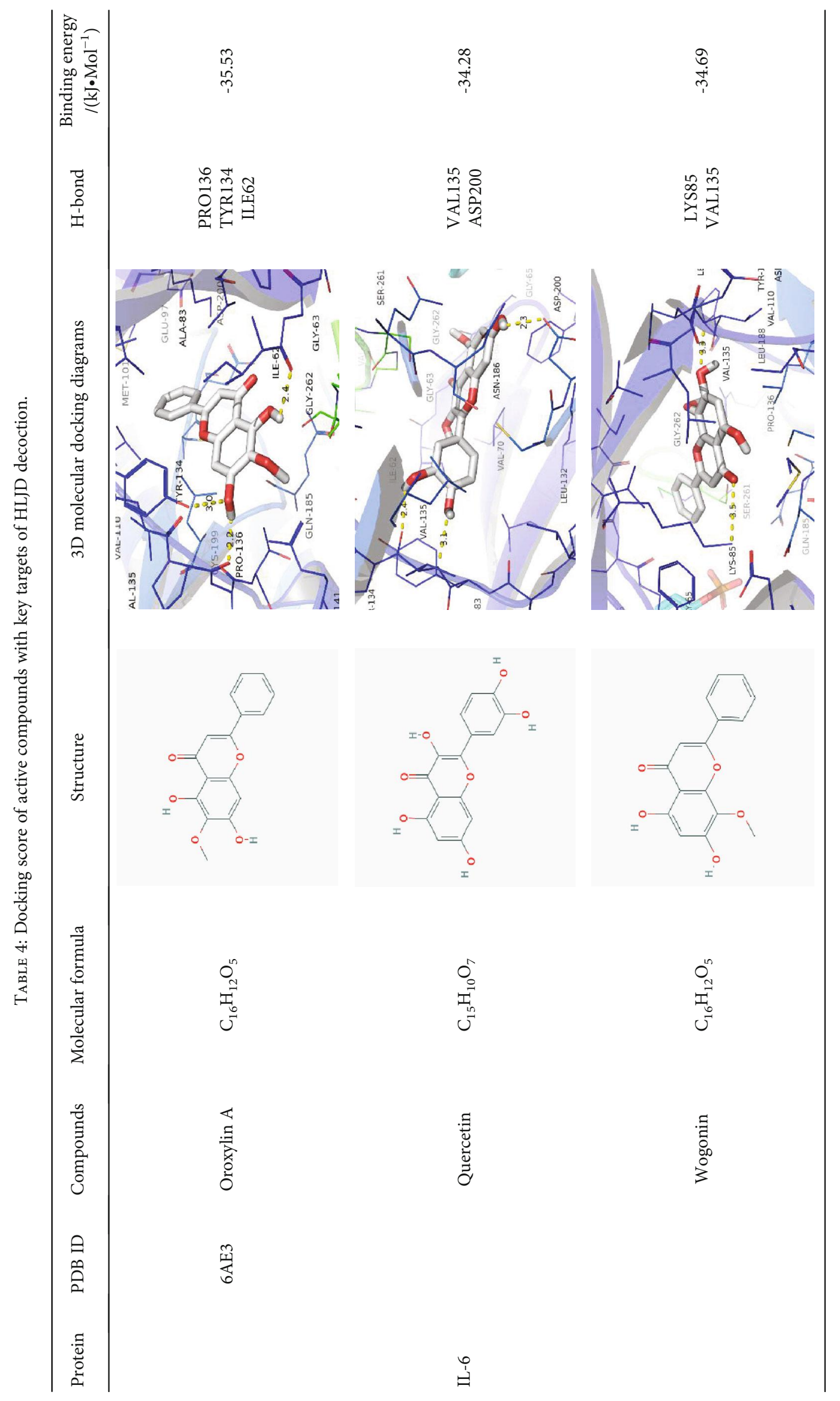




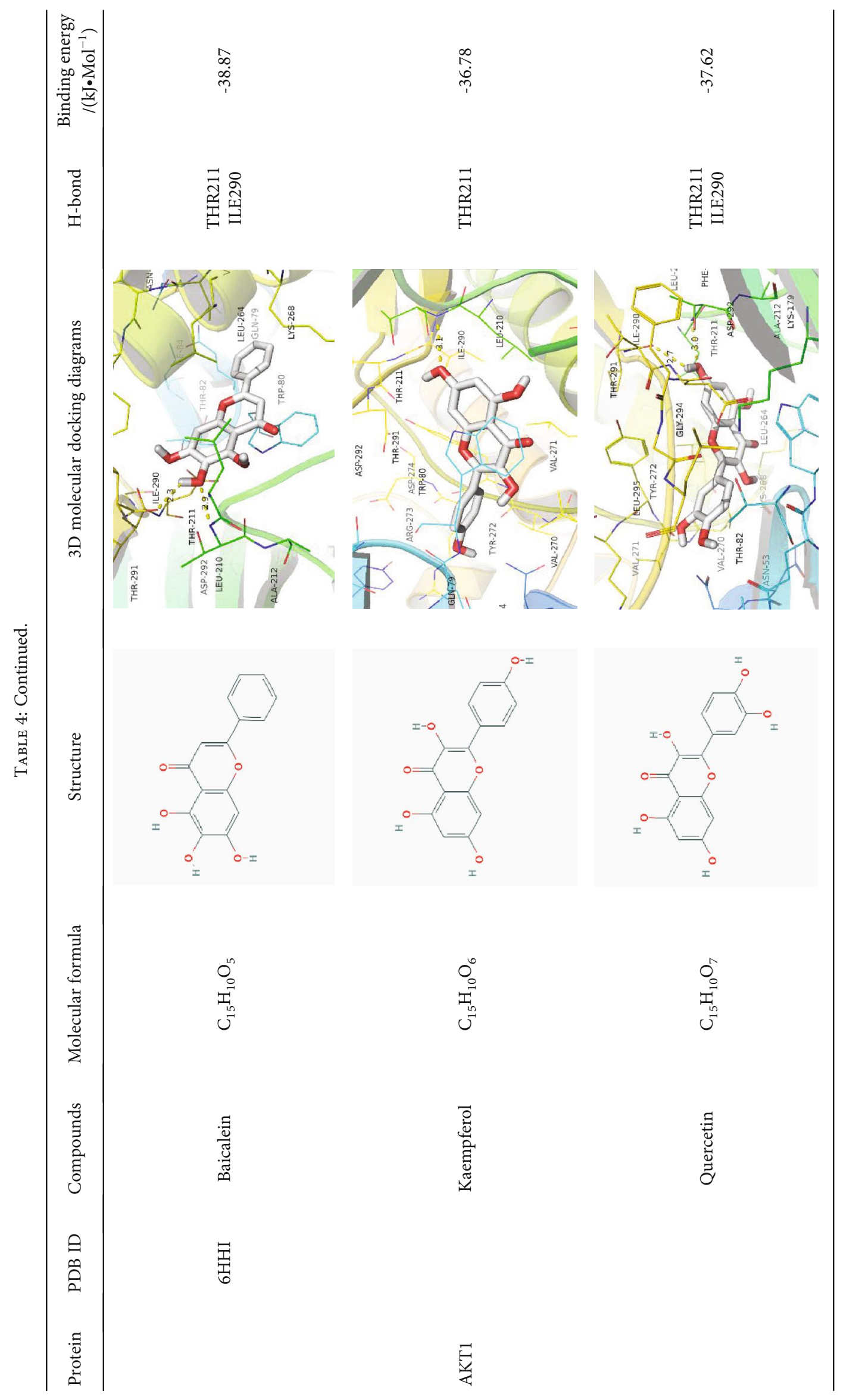




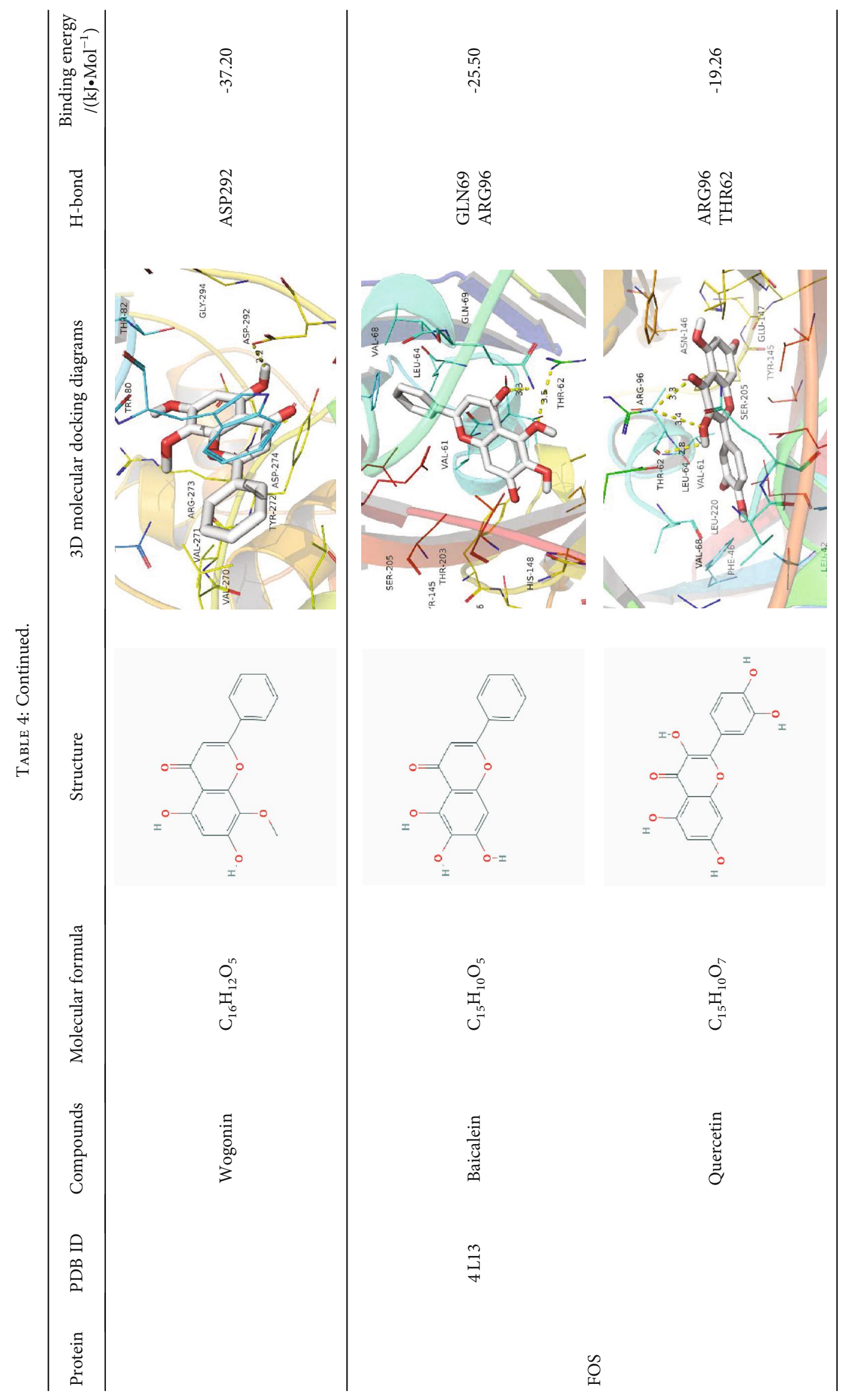






FIGURE 10: Decoction-compound-target-pathway network (Crimson indicates the HLJD decoction; red indicates the herbs; yellow indicates the compounds; green indicates the key targets; purple indicates the pathways).

\section{Conclusion}

In conclusion, this comprehensive bioinformatic analysis provides numerous testable hypotheses about the molecular mechanisms of HLJDD formula for the treatment of T2DM as well as diabetic complications (cognitive dysfunction, vascular disease, and cardiomyopathy) and predicted that quercetin, baicalein, wogonin, kaempferol, and oroxylin A were potential active ingredients of HLJDD, which acts on key genes such as AKT1, IL-6, and FOS to regulate many biological processes (signaling transduction, inflammatory response, apoptotic process, vascular processes, etc.) and pathways (AGE-RAGE signaling pathway in diabetic, IL-17signaling pathway, TNF signaling pathway, Toll-like receptors signaling pathway, type II diabetes, endocrine resistance, insulin resistance signaling pathways, etc.). It also should be noted that HLJDD formula possible exhibits antitumor effect by regulating multiple tumorrelated pathways. The prediction results provided the theoretic elucidation of the ameliorative effect of HLJDD against T2DM and might facilitate the development of HLJDD or its active compounds as alternative therapy for T2DM.

\section{Data Availability}

The data used to support the findings of this study are available from the corresponding author upon request.

\section{Conflicts of Interest}

The authors declare that they have no conflicts of interest.

\section{Authors' Contributions}

BY designed the study and wrote the original draft. YM B and YQ X collected the data and wrote the original draft. GJ F administrated the project and edited the manuscript. All authors read and approved the manuscript.

\section{Acknowledgments}

This study was partially supported by the Chinese Government, Ministry of Science and Technology of the People's Republic of China through the National Science and Technology Support Program (grant number 2015BAI04B09).

\section{References}

[1] R. A. DeFronzo, "Type 2 diabetes mellitus," Nature Reviews Disease Primers, vol. 1, no. 1, article 15019, 2015.

[2] J. E. Shaw, R. A. Sicree, and P. Z. Zimmet, "Global estimates of the prevalence of diabetes for 2010 and 2030," Diabetes Research and Clinical Practice, vol. 87, no. 1, pp. 4-14, 2010.

[3] Y. Zheng, S. H. Ley, and F. B. Hu, "Global aetiology and epidemiology of type 2 diabetes mellitus and its complications," Nature Reviews Endocrinology, vol. 14, no. 2, pp. 88-98, 2018.

[4] Global Burden of Disease Study 2013 Collaborators, "Global, regional, and national incidence, prevalence, and years lived with disability for 301 acute and chronic diseases and injuries in 188 countries, 1990-2013: a systematic analysis for the Global Burden of Disease Study 2013," The Lancet, vol. 386, no. 9995, pp. 743-800, 2015.

[5] T.-t. Guo, "Efficacy of single administration of Huanglian Jiedu Tang and combined administration with basic medicine for type 2 diabetes of meta-analysis," Diabetes New World, vol. 22, no. 19, pp. 53-54, 2019. 
[6] Y. Chen, Q. Wu, Y. Liu, and S. Xiong, "Efficacy of single administration of Huanglian Jiedu Tang and combined administration with basic medicine for type 2 diabetes of meta-analysis," Chinese Journal of Experimental Traditional Medical Formulae., vol. 24, no. 10, pp. 212-220, 2018.

[7] X. J. Zhang, Y. X. Deng, Q. Z. Shi, M. Y. He, B. Chen, and X. M. Qiu, "Hypolipidemic effect of the Chinese polyherbal Huanglian Jiedu decoction in type 2 diabetic rats and its possible mechanism," Phytomedicine, vol. 21, no. 5, pp. 615-623, 2014.

[8] S.-K. Yan, J. Zhao, S.-S. Dou, P. Jiang, R.-H. Liu, and W.D. Zhang, "Methodology of modernization research in traditional Chinese medicine based on systems biology and network biology," Chinese Journal of Natural Medicines, vol. 7, no. 4, pp. 249-259, 2009.

[9] L. Breuza, S. Poux, A. Estreicher et al., "The UniProtKB guide to the human proteome," Database, vol. 2016, article bav120, 2016.

[10] P. Jain, S. Vig, M. Datta et al., "Systems biology approach reveals genome to phenome correlation in type 2 diabetes," PLoS One, vol. 8, no. 1, article e53522, 2013.

[11] O. Trott and A. J. Olson, "AutoDock Vina: improving the speed and accuracy of docking with a new scoring function, efficient optimization, and multithreading," Journal of Computational Chemistry, vol. 31, no. 2, pp. 455-461, 2010.

[12] T. Gaillard, "Evaluation of AutoDock and AutoDock Vina on the CASF-2013 benchmark," Journal of Chemical Information and Modeling, vol. 58, no. 8, pp. 1697-1706, 2018.

[13] J. Zhang, H. Cao, J. Xu, Y. Han, S. Gong, T. Zhang et al., "Expression of bitter taste of Chinese materia medica and its application in clinical compatibility," Chinese Traditional and Herbal Drugs, vol. 47, no. 2, pp. 187-193, 2016.

[14] X. Liu, Z. Bao, X. Zhuang, L. Que, and C. Tian, "Discussion on strengthening yin of Chinese herbs with bitter-flavor-clinical traditional Chinese pharmacology noun terminology standardization research," China Journal of Chinese Materia Medica, vol. 38, no. 20, pp. 3591-3594, 2013.

[15] B. Li, W. Wu, S. Xie, X. Zhang, and F. Wang, "Effects of Huanglian Jiedu decoction on learning and memory ability and tau protein phosphorylation pathway of Alzheimer-like in rats with type 2 diabetes," China Journal of Traditional Chinese Medicine and Pharmacy, vol. 30, no. 5, pp. 14211425, 2015.

[16] Y. Xiao, F. Lu, L. Xu, S. Leng, and K. Wang, "Protective effects of Huanglian Jiedu decoction on vascular endothelial function in type 2 diabetic rats," China Journal of Chinese Materia Medica., vol. 30, no. 22, pp. 47-50, 2005.

[17] S. Leng, F. Lu, Q. Tu, L. Xu, M. Yang, and K. Wnag, "Effects of Huang Lian Jie Du decoction on blood glucose and lipids metabolisms in type II diabetic rats," Chinese Journal of Basic Medicine in Traditional Chinese Medicine, vol. 9, no. 4, pp. 43-45, 2003.

[18] W. Wu, S. Xie, B. Li, B. Li, and X. Liu, "Effects of Huanglian Jiedu decoction on the glucosylation of glucose transporter protein 3 and O-GlcNAc of tau protein in the brain of rats with type 2 diabetes," China Journal of Traditional Chinese Medicine and Pharmacy, vol. 32, no. 6, pp. 2706-2709, 2017.

[19] H. Alkhalidy, W. Moore, Y. Zhang et al., "Small molecule kaempferol promotes insulin sensitivity and preserved Pancreatic $\beta$-Cell mass in middle-aged obese diabetic mice," Journal of Diabetes Research, vol. 2015, Article ID 532984, 14 pages, 2015.
[20] H. Jiang, Y. Yamashita, A. Nakamura, K. Croft, and H. Ashida, "Quercetin and its metabolite isorhamnetin promote glucose uptake through different signalling pathways in myotubes," Scientific Reports, vol. 9, no. 1, article 2690, 2019.

[21] Q. U. Ahmed, M. N. Sarian, S. Z. Mat So'ad et al., "Methylation and acetylation enhanced the antidiabetic activity of some selected flavonoids: in vitro, molecular modelling and structure activity relationship-based study," Biomolecules, vol. 8, no. 4, article 149, 2018.

[22] H. Alkhalidy, W. Moore, A. Wang et al., "Kaempferol ameliorates hyperglycemia through suppressing hepatic gluconeogenesis and enhancing hepatic insulin sensitivity in dietinduced obese mice," The Journal of Nutritional Biochemistry, vol. 58, pp. 90-101, 2018.

[23] K. Suchal, S. Malik, S. I. Khan et al., "Molecular pathways involved in the amelioration of myocardial injury in diabetic rats by kaempferol," International Journal of Molecular Sciences, vol. 18, no. 5, article 1001, 2017.

[24] Z. Yang, W. Huang, J. Zhang, M. Xie, and X. Wang, "Baicalein improves glucose metabolism in insulin resistant HepG2 cells," European Journal of Pharmacology, vol. 854, pp. 187193, 2019.

[25] L. Lu, Q. Guo, and L. Zhao, "Overview of oroxylin A: a promising flavonoid compound," Phytotherapy Research, vol. 30, no. 11, pp. 1765-1774, 2016.

[26] T. Tomita, "Apoptosis in pancreatic $\beta$-islet cells in type 2 diabetes," Bosnian Journal of Basic Medical Sciences, vol. 16, no. 3, pp. 162-179, 2015.

[27] R. M. Abdel-Megeed, S. A. el Newary, M. O. Kadry et al., "Hyssopus officinalis exerts hypoglycemic effects on streptozotocin-induced diabetic rats via modulating GSK$3 \beta$, C-fos, NF- $\kappa \mathrm{B}, A B C A 1$ and $A B G A 1$ gene expression," Journal of Diabetes and Metabolic Disorders, vol. 19, no. 1, pp. 483-491, 2020.

[28] M. Prause, C. M. Mayer, C. Brorsson et al., "JNK1 deficient insulin-producing cells are protected against interleukin- $1 \beta$ induced apoptosis associated with abrogated Myc expression," Journal of Diabetes Research, vol. 2016, Article ID 1312705, 15 pages, 2016.

[29] Z. Rui, The research of apoptosis and function of the rat islet which was transfected Akt1 gene via adenovirus vector, China Medical University, 2007.

[30] Z.-Y. Xu, Study on DNA methylation alteration of vascularsmooth muscle cell proliferation related genesin type 2 diabetic macrovascular complications, Huazhong University of Science and Technology, 2014.

[31] G. Daniele, R. Guardado Mendoza, D. Winnier et al., "The inflammatory status score including IL- 6 , TNF- $\alpha$, osteopontin, fractalkine, MCP-1 and adiponectin underlies whole-body insulin resistance and hyperglycemia in type 2 diabetes mellitus," Acta diabetologica, vol. 51, no. 1, pp. 123-131, 2014.

[32] R. Wu, X. Liu, J. Yin et al., "IL-6 receptor blockade ameliorates diabetic nephropathy via inhibiting inflammasome in mice," Metabolism, vol. 83, pp. 18-24, 2018.

[33] Y. L. Konheim and J. K. Wolford, "Association of a promoter variant in the inducible cyclooxygenase-2 gene (PTGS2) with type-2 diabetes mellitus in Pima Indians," Human Genetics, vol. 113, no. 5, pp. 377-381, 2003.

[34] Y. Luo, J. Luo, and H. Peng, "Associations between genetic polymorphisms in the VEGFA, ACE, and SOD2 Genes and susceptibility to diabetic nephropathy in the Han Chinese," 
Genetic Testing and Molecular Biomarkers, vol. 23, no. 9, pp. 644-651, 2019.

[35] Z. Wang, L. Chang, J. Wu et al., "A novel rhynchophylline ana$\log , \mathrm{Y} 396$, inhibits endothelial dysfunction induced by oxidative stress in diabetes through epidermal growth factor receptor," Antioxidants \& Redox Signaling, vol. 32, no. 11, pp. 743-765, 2020.

[36] R. Adela, P. N. C. Reddy, T. S. Ghosh et al., "Serum protein signature of coronary artery disease in type 2 diabetes mellitus," Journal of Translational Medicine, vol. 17, no. 1, article 17, 2019.

[37] D. Chawla, S. Bansal, B. D. Banerjee, S. V. Madhu, O. P. Kalra, and A. K. Tripathi, "Role of advanced glycation end product (AGE)-induced receptor (RAGE) expression in diabetic vascular complications," Microvascular Research, vol. 95, pp. 1-6, 2014.

[38] A. Csiszar and Z. Ungvari, "Endothelial dysfunction and vascular inflammation in type 2 diabetes: interaction of AGE/RAGE and TNF- $\alpha$ signaling," American Journal of Physiology Heart and Circulatory Physiology, vol. 295, no. 2, pp. H475H476, 2008.

[39] Y. Deng, L. He, S. Wan et al.et al., "Effects of Huanglian Jiedu decoction on formation of advanced glycation end products in vivo and in vitro," Chinese Traditional and Herbal Drugs, vol. 42, no. 1, pp. 130-133, 2011.

[40] P. K. Patibandla, A. J. Rogers, G. A. Giridharan, M. A. Pallero, J. E. Murphy-Ullrich, and P. Sethu, "Hyperglycemic arterial disturbed flow niche as an in vitro model of atherosclerosis," Analytical Chemistry, vol. 86, no. 21, pp. 10948-10954, 2014.

[41] R. Simo, A. Ciudin, O. Simo-Servat, and C. Hernandez, "Cognitive impairment and dementia: a new emerging complication of type 2 diabetes-the diabetologist's perspective," Acta diabetologica, vol. 54, no. 5, pp. 417-424, 2017.

[42] C. T. Kodl and E. R. Seaquist, "Cognitive dysfunction and diabetes mellitus," Endocrine Reviews, vol. 29, no. 4, pp. 494-511, 2008.

[43] L. Sun, L. Liu, H. Zhu, B. Zhu, and Q. Zhang, "Network pharmacology-based study on intervention mechanism of Huanglian Jiedu decoction in the treatment of Alzheimer's disease," Acta Pharmaceutica Sinica, vol. 52, no. 8, pp. 12681275, 2017.

[44] Y. Zhou, Y. Zhao, H. Xie, Y. Wang, L. Liu, and X. Yan, “Alteration in amyloid $\beta 42$, phosphorylated tau protein, interleukin 6 , and acetylcholine during diabetes-accelerated memory dysfunction in diabetic rats: correlation of amyloid $\beta 42$ with changes in glucose metabolism," Behavioral and Brain Functions, vol. 11, no. 1, article 24, 2015.

[45] M. Dewenter, A. von der Lieth, H. A. Katus, and J. Backs, "Calcium signaling and transcriptional regulation in cardiomyocytes," Circulation Research, vol. 121, no. 8, pp. 1000-1020, 2017.

[46] J. Wang, Z. Tang, Y. Zhang et al., "Matrine alleviates AGEsinduced cardiac dysfunctions by attenuating calcium overload via reducing ryanodine receptor 2 activity," European Journal of Pharmacology, vol. 842, pp. 118-124, 2019.

[47] G. Shlomai, B. Neel, D. LeRoith, and E. J. Gallagher, "Type 2 diabetes mellitus and cancer: the role of pharmacotherapy," Journal of Clinical Oncology, vol. 34, no. 35, pp. 4261-4269, 2016. 